\title{
Lipomyelomeningocele: A Cause of Closed Dysraphism with Fatty Contingent. Case Report and Review of the Literature
}

\author{
I. Zouita ${ }^{1}$, F. Z. Abakka ${ }^{1 *}$, J. F. Adjimabou ${ }^{1}$, D. Basraoui ${ }^{1}$, H. Jalal $^{1}$
}

${ }^{1}$ Radiology Mother Child Department, CHU Mohammed VI, University Cadi Ayad, Marrakech, Morocco

DOI: $10.36347 /$ simcr.2021.v09i04.041

| Received: 17.03.2021 | Accepted: 26.04.2021 | Published: 30.04.2021

*Corresponding author: F. Z. Abakka

Abstract

Case Report

Lumbosacral lipomasrepresent the most common forms of occult spinal dysraphism. Lipomyelomeningocele is a rare birth defect of the spine. It's a lipoma that is tightly attached to the dorsal surface of a neural placode and extend dorsally through spina bifida to be continuous with subcutaneous fat. MRI plays an essential role in the diagnosis and treatment. We report the case of an 18-month-old infant in whom we detected a lipomyelomeningocele during the exploration of scoliosis.

Keywords: Lipoma, imaging, lipomyelomeningocele.

Copyright $\odot 2021$ The Author(s): This is an open-access article distributed under the terms of the Creative Commons Attribution 4.0 International License (CC BY-NC 4.0) which permits unrestricted use, distribution, and reproduction in any medium for non-commercial use provided the original author and source are credited.

\section{INTRODUCTION}

Lumbosacral lipomas are the term under which spinal lipomas are grouped, in particular lipomas of the medullary cone and terminal filum. They represent the most common forms of occult spinal dysraphism (spina bifida occulta), which are a large group of malformations loosely grouped together by their closed character, ie covered by the skin [1]. Lipomyelomeningocele is a type of occult spinal dysraphism consisting of the presence of lipomatous tissue attached to the spinal cord through a defect in the spine containing the meninges or spinal cord and forming a posterior mass under the skin [2]. We report the case of an 18-month-old infant in whom we detected a lipomyelomeningocele during the exploration of scoliosis.

\section{Case Report}

This is an 18 months old infant with a spinal deformity that progresses from birth. An x-ray of the dorsolumbar spine was performed showing dorsolumbar scoliosis.

An MRI was then performed (Fig-1), showing a bony defect in the posterior arches of the lumbosacral region lateralized to the right and extending for over 4.5 $\mathrm{cm}$ from L4 to S1 with a large hernia of the dural sac containing cerebrospinal fluid and the nerve roots of the cauda equinawith individualization within it of a subcutaneous compressive fatty formation infiltrating medially and pushing forward the right psoas muscle; appearing hyperintense in $\mathrm{T} 1$ weighted sequences and fading on FATSAT sequences without contrast enhancement.

This mass infiltrates the canal, attracting the roots of the cauda equina and pushing back the medullary cone, revealing syringomyelic cavities that appear hyperintense in T1 and hypointense in T2. It is associated with an expansion of the peri-medullary spaces at its level, responsible for an enlargement of the medullary canal.

The spinal scan revealed dorsolumbar scoliosis associated with vertebral malformations associating a rotational component, tiered hemivertebraes from D11 to L1 and fusion of the vertebral bodies from L1 and L2.

In light of thoses findings, the diagnosis of lipomyelomeningocele associated with dorsolumbar scoliosis and vertebral malformations was retained. 

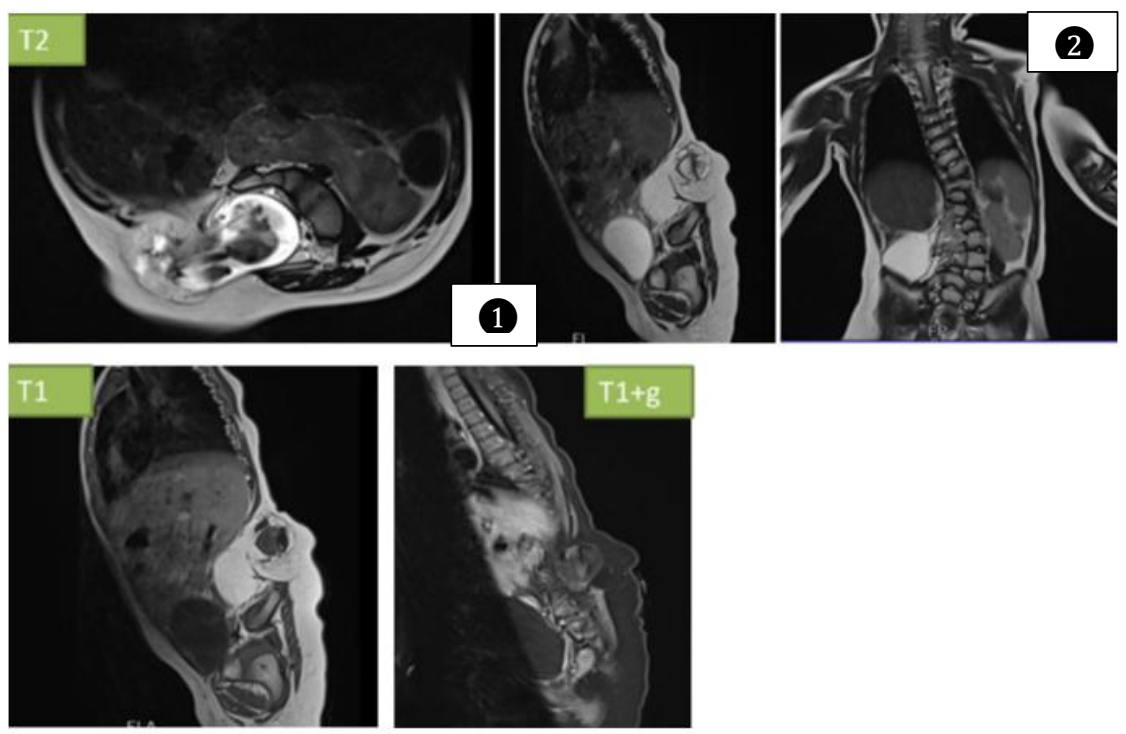

Fig-1: Medullary MRI with T1 / T2 and T1 sequence with Gadolinium injection:

1 Bone defect of the posterior arches of the lumbosacral region extended from L4 to S1 with a large hernia of the dural sac containing cerebrospinal fluid, the nerve roots of the cauda equina and an infiltrating subcutaneous compressive fatty formation

2 Dorsolumbar scoliosis associated with vertebral malformations associating a rotational component, blind tiered hemivertebrae from D11 to L1 and fusion of the vertebral bodies of L1 and L2.

\section{DiSCUSSION}

Lipomyelomeningocele is a rare birth defect of the spine. The most common clinical presentation is the presence of a lipomatous-looking mass located in the midline in the lumbosacral region [2]. However, this mass can be associated with other clinical signs of the skin such as tufts of hair, lumbosacral dimples, or cutaneous hemangiomas. Other no less frequent signs can also be objectified, in particular a motor deficit, sensory disorders, spasticity, urinary and urorectal sphincter disorders or orthopedic deformities [3]. The progressive nature of the onset of these symptoms is suggestive [4]. In our case, it was a spinal deformity showing then the importance of thinking about this diagnosis in the face of any closed spinal deformity.

In some series like that of Kanev et al., [2], intestinal and bladder functions deteriorate before motor or sensory function. In this series, patients presented with complete bowel paralysis as well as motor impairment of the bladder before the onset of motor and sensory loss on physical examination. In our case, these signs were not found, thus testifying to a fairly early diagnosis, and justifying the need to explore any occult dysraphism objectified from birth to avoid the development of complications that may be irreversible.

In the literature, lipomyelomeningocele appears to affect more females than males [4]. Our patient being male and given that the diagnosis was made at 18 months and that there is no major neurological complication, the hypothesis according to which the pathology is more benign in the male sex should be verified and would require a study on a much larger sample.

$$
\text { Classically, } 3 \text { categories of }
$$

lipomyelomeningocele are described according to the location: dorsal, transitional and caudal [2]. In our patient, it was a caudal type. However, cervical localization has been described by Heidari et al., [4].

MRI plays an essential role in the diagnosis and treatment of lipomyelomeningocele. MRI findings include expansion of the spinal canal and subarachnoid spaces, dorsal extension of the spinal cord and dura through spinal dysraphism. The stretched and deformed character of the marrow which faces the lipoma is a common sign. The $\mathrm{T} 1$ and $\mathrm{T} 2$ sequences are extremely essential, in particular for surgical management. They make it possible to determine the neurosurgical anatomy of the lesion and its relationship with neighboring structures. MRI also helps in detecting associated malformations of the spinal cord and the presence of syringomyelic cavities. The threedimensional approach is very useful because it offers flexibility for performing multi-planar reconstructions, especially in patients with spinal deformity as is the case in our observation [1].

Since the natural progression of lipomyelomeningocele is the progression to neurological deficits and/or urological disorders, prophylactic surgery is recommended. The goals of the surgery are to eliminate fatty masses in order to relieve the effect of attachment on the spine to preserve neuronal tissues [2]. 


\section{CONCLUSION}

Lipomyelomeningocele is a rare condition. It's a lipoma that is tightly attached to the dorsal surface of a neural placode and extend dorsally through spina bifida to be continuous with subcutaneous fat. Imaging, in particular MRI, plays a major role in topographic diagnosis as well as in the analysis of relationships with neighboring structures in order to guide therapeutic management, which in general is surgical.

\section{Conflict of Interest: No Conflict of Interest.}

\section{REFERENCES}

1. Morioka T, Murakami N, Shimogawa T, Mukae N, Hashiguchi K, Suzuki SO, Iihara K. Neurosurgical management and pathology of lumbosacral lipomas with tethered cord. Neuropathology. 2017 Oct;37(5):385-92.

2. Niryana W, Rahayu NS, Widyadharma IP. Lipomyelomeningocele Lumbosacral: A Case Report. International Journal of Science and Research (IJSR). 2015;6:391.

3. Kang JK, Lee KS, Jeun SS, Kang SG, Lee IW, Yoon KJ, Ha SS. Tethered cord syndrome; surgical indication, technique and outcome. Journal of Korean Neurosurgical Society. 2007;42(2):77-82.

4. Heidari SV, Mollahoseini R, Ghandehari H, Farhadi E, Abbasi F, Asaadi S, Soulat M, Bavand K, Nejat F. Cervical Lipomyelomeningocele Presenting with Progressive Motor Deficit: A Case Report and Review of the Literature. Pediatric neurosurgery. 2020;55(1):58-61. 